\title{
Networked Bridge Deflection Monitor Platform Design
}

\author{
Dandan Zhang ${ }^{1, a}$, Hongwei Zhao ${ }^{2, b}$ and Yunfeng Song ${ }^{2, c}$ \\ ${ }^{1}$ School of Electronic \&Control Engineering Chang'an University, Shaanxi 710064, China \\ ${ }^{2}$ Wei-Nan Highway quality supervisory station, Wei'nan XXXXXX, Shaanxi 714000, China \\ ${ }^{3}$ Wei-Nan Highway quality supervisory station, Wei'nan XXXXXX, Shaanxi 714000, China \\ a2507477017@qq.com, ${ }^{\text {b } 1716377527 @ q q . c o m, ~}{ }^{\mathrm{c}} 738814440 @ q q . c o m$
}

Keywords: Bridge defection, Baidu map, Mysql database ,Web server, Android app.

\begin{abstract}
To facilitate bridge managers to monitor real time bridge safety level and then conduct in-time handling and reporting, this study utilized real time bridge deflection data from MCU and informational circumstance of a combination of mobile terminals and computers to establish a database of basic bridge information and real time data charts of each bridge deflection, then, through Baidu map, realized bridge information labeling, bridge searching, real time bridge deflection data displaying and designing of a monitor platforming combining bridge danger grades and bridge label variations. After that the study built an independent information extracting and exchanging platform based on one module and two platforms to provide users with bridges' real time changing situation as well as alarms. In addition, the paper developed a highly practical full-set monitoring plan which possesses strong referential implications for bridge managers to monitor their bridges and to make concrete maintenance plans in time.
\end{abstract}

\section{Platform Introduction}

In parallel with the development of transportation industry, bridge managing and maintenance work has been increasingly heavy. Moreover, under the effects of climate, oxidation, erosion, aging, engineering construction, over-ranging vehicle flow-rate and vehicle overloading, bridge strength and stiffness decrease as time pass, and components become more fragile to damage after long terms of static and live loading, which not only affects pedestrian safety, but also shortens bridges' service lives. For recent years internet information technology has been flourishing in a variety of areas, therefore combining bridge testing results and Android application to build an online bridge deflection monitor platform will have crucial implications on informational treatment of bridges with hidden dangers and real time monitor of bridge situations.

This platform is a mobile terminal that utilizes data transfer unit (DTU) to mainly receive MUC real time deflection data, bridge map and specific data of bridge deflection variation, and simultaneously exchanges information with Web platform to provide users with real time bridge information anytime and anywhere. Users could find out all bridge names as well as icons indication safety grade, search and locate a specific bridge and click the bridge icons to see detailed deflection data.

This system consists of multiple parts: DTU data receiving module, Android platform design and Web platform design (see Fig 1). DTU data receiving module and Web platform carry out database operation with a database source; Web platform communicates with Android platform to conduct data exchange.

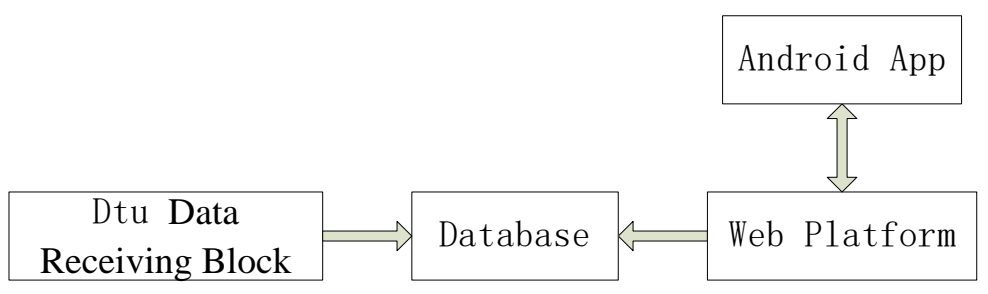

Fig. 1 System structure diagram 


\section{DTU Data Receiving Module}

DTU data receiving module utilizes ServerScoket to realize communications between customers and specific server ports. Server terminal receives requests from different customers, identifies these customers then establishes threads to build connections, receive customers data, differentiate different data types and finally create database or insert operations to the data.

\subsection{Receiving and Unsealing JSON Data}

Communications between customers and server terminals are sealed though JSON, therefore it is necessary to unseal the data after receiving from customers. The unsealing process includes introduction of Jar package, adding citations of that Jar package to the project and application of JsonUtil.toObject() method to convert JSON formate data to predefined Java Bean objects, then acquiring internal sub-objects or sealed data and finally acquiring internal data after a serials of unsealing steps.

\subsection{Building Connections to Database}

The next step after unsealing JSON data is to conduct agreement content judgement based on different raw numbers received, and then, according to different judgment results, to make following response: 1. Unsealing first raw of data and acquiring basic bridge information customers just sent, including bridge name, longitude, latitude and height, then creating corresponding database charts in database . 2. Unsealing received data, acquiring number of bridge registration, assembling into a database chart name corresponding to the bridge, then creating database link of the specific bridge. 3 . In a circulation, unsealing each raw of data, cutting data on the basis of database links generated in previous two steps, then storing in specific database charts.

\subsection{Database Operation}

After data unsealing step is to build connections with database and to conduct database operation. There are many ways in building database connection, and this platform chose self constructed connection pool with an initially set connection number as 5. As the amount of customer access exceeds 5, the connection pool will build new connections automatically. Embedded in this connection pool are several methods of acquiring connections, acquiring data source and returning database connection as well as language executors after usage. The platform utilizes customer-friendly thoughts, seals both databased connections customers need when visiting databases and customers returned after stopping visiting into MyDataSource category for convenient building and extracting.

Information processing of DTU includes classification of unsealed data and relevant operations in well built DUT database. Operations could be classified into totally two categories, first of which is to create database charts and add basic information, the other is to add, delete, modify or check database charts.

\section{Android Platform}

The Android platform consists of five modules: bridge registration module, map environment construction module, bridge label management module, bridge searching module and bridge deflection indication module (see Fig 2).Android terminal loads Baidu maps when it gets online, communicates with Web server to acquire App bridge data and labels bridge names and icons on map. Users could click searching box to search for bridge of interest and click bridge icon to check real time deflection changing curves of specific bridges. 


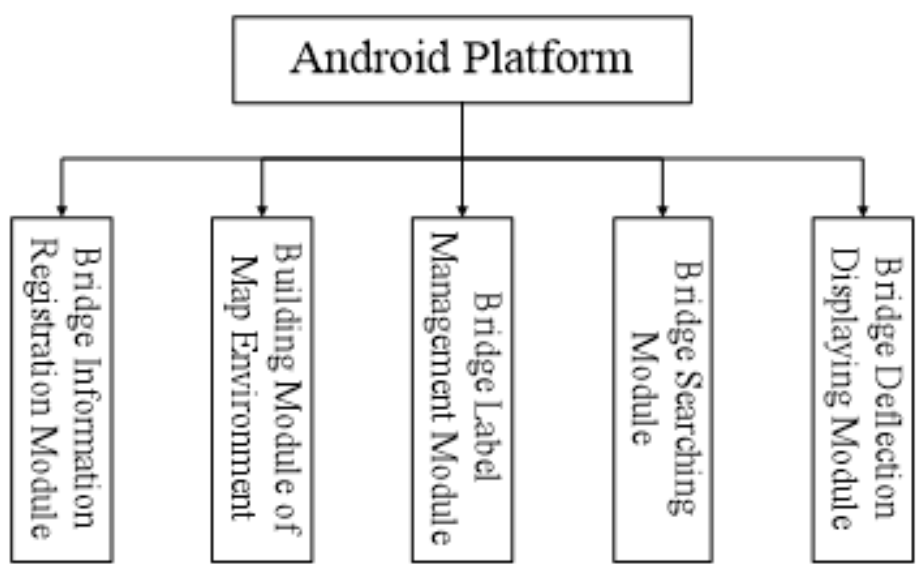

Fig. 2 Five modules of the Android platform

\subsection{Bridge Registration Module}

In bridge registration module, first time customers need to register bridges, including bridge name, its longitude and latitude. They will not see the registration page next time they open the app but will directly enter map pages. This system uses SharedPreferences, a lightweight storage category in Android platform, to store a variable isFirst in order to judge whether it is the first time a customer open the app or not. The value of isFirst will be changed to FALSE after that for later time judgement use.

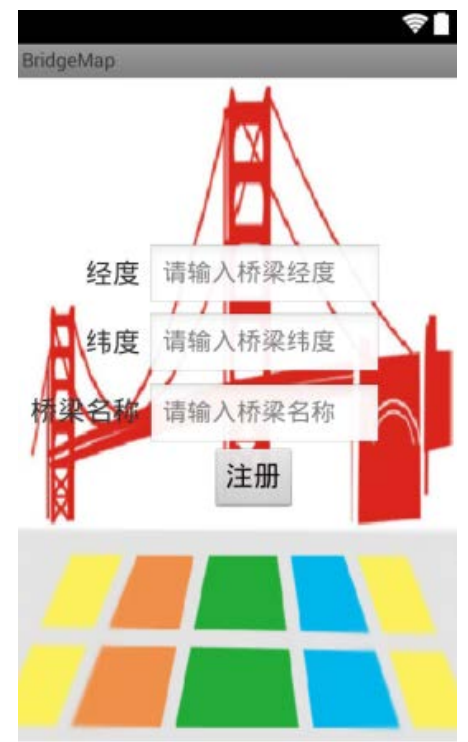

Fig. 3 Registration interface

\subsection{Map Environment Construction Module}

Map environment construction module is mainly responsible for the configuration and displaying of basic map information. It utilized Baidu map SDK provided by Baidu, applied API to set up basic functions such as map center, scale, etc. This module uses self-defined zoom controls to beautify the entire page and to edit map page and utilizes Baidu map API to set up map script, icon clicking events and searching box clicking events.

\subsection{Bridge Label Management Module}

Bridge label management module contains two parts: creating bridge labels on map and changing labels during bridge situation changing process.

Bridge label on initially built map is created in following procedure: terminals communities with Web server and acquires all basic bridge information charts from database, then unseals JSON data, creates JAVA Bean object, after using subject acquired registration information of each bridge, seals registration icon category TextMarker, in circulation creates target objects and finally realizes map icon labeling. 
Bridge situation may present three states: safety state, alarming state and danger state. Specific evaluating criteria are shown below in Table 1. The icon is set to be green in safe state, yellow in alarming state and red flashing in danger state.

Table 1 Bridge Situation and Evaluating Criteria

\begin{tabular}{cc}
\hline Bridge State & Criteria (for each 300 data sources) \\
\hline Safety State & $<5$ times exceeding alarming line, $<3$ times exceeding danger line \\
Alarming State & $<20$ times exceeding alarming line, $<10$ times exceeding danger line \\
Danger State & $>30$ times exceeding alarming line, $>15$ times exceeding danger line
\end{tabular}

\subsection{Bridge Searching Module}

In bridge searching module, users click searching box, then enter searching page and input name of the bridge they want to search. After that, this module will display all bridge names with the same first letter to reduce users' searching efforts. When users click the appeared bridge names, the app will convert back to map pages and show a dialog box asking users whether to change default map center or not; if users click YES, the default map center changes accordingly, and a NO will lead to opposite result. The module utilizes AutoCompleteTextView control in real time displaying all bridge names, then establish a data adaptor for AutoCompleteTextView control, fulfills it with a set of all bridges names extracted from Android internal database Sqlite, uses SharedPreferences category to store longitude and latitude of the bridge under clicking, converts pages, then compares the longitude and latitude of the bridge under clicking to those of default map display center to realize the changing of default map display center.

\subsection{Real Time Bridge Deflection Displaying Module}

As users click bridge icon, they will enter real time bridge deflection display page. This page presents bridge name and real time deflection changing curve. Bridge name is extracted from local Sqlite database and displayed. Real time bridge deflection curve is created in following procedure: terminal gets online and visit server database, creates methods, makes real time curve, then design a repetitive data extracting operation in every 10 seconds and accordingly remake deflection curve.

\section{Web Platform}

Web platform functions mainly on building a database server platform. It receive users' requests, differentiates users' operation, conducts corresponding database operation and returns user data. Server terminal could use IIS, Apache, Tomcat, etc., and it depends on users' familiarization level to Web service environment framework to determine different Web service operation environments. This platform utilizes Tomcat for Web service, Mysql for database support and JSON for Web server terminal and data connecting port to Android terminal.

JSON is a lightweight data exchange formate. It could convert a group of data to a string of characters, then readily transfer this string among servers, or transfer it from Web server terminal to asynchronous apps. In addition, Android provides fantastic technique support for JSON.

\section{Conclusion}

In the age of mobile internet, applying internet technology on real time monitor of bridge safety will infill new energies to real time bridge deflection variation monitor technology. The bridge deflection variation monitor platform has various advantages, such as in time responding, low operation cost, good stability, high maintainability, etc. It could realize real time monitor of bridge operation and then predict prospect development in a easy process, which facilitates users to monitor and establish reasonable, active and preventive maintenance plans. In this way, it could help users control bridge situations and elongate bridges' service lives in a large degree, and therefore effectively reducing operation and maintenance cost of bridges during the entire service lives. 


\section{Acknowledgments}

The authors thanks the editors and the anonymous reviewers for their valuable comments that helped to improve the paper. The work was supported by the Science and technology project of shaanxi provincial transportation department(No.17-16K,17-33T).

\section{References}

[1] Gong Chengying, Xing Jinghong, Gu Yinbao, et al. 2013. Data communication of Android mobile terminal and PHP and MySQL based on JSON[J], Engineering Meters and Automatic Facilities, Vol. 1(63-65).

[2] Xu Li-xian. 2014. Research on The Application Technology based on Baidu Map in Android system. Vol. 9(86-87).

[3] Wang Hongsong, Zhouhaiyan. 2012. Development of Tourism Geographic Information System Based on Baidu Map AP. Vol. 23(60-63).

[4] Du Chuanming. 2011. Application of BaiDu Map API in Small Geographic Information System[J]. Geometics and Spatial Information Technology, Vol. 2.

[5] Zhou Tong, Long Yi. 2012. Review about Recently Development of Mobile Map and Internet Map in China[J], Geology and Geoinformatics, Vol. 5(1-5).

[6] Yang Yanmei, Wang Genze. 2007. Research on Theory Basic of Mobile Map[J], Sichuan Geometics, Vol. 4(159-163).

[7] Wang Guilin, Yang Kun, Yang Yang, et al. 2015. Design and Implementation of GIS Combined with Baidu Map Application System Contruction, Bulletin of Science and Technology, Vol. 31(58-61). 